\title{
A NEAR-INFRARED STUDY OF THE HIGHLY-OBSCURED ACTIVE STAR-FORMING REGION W51B
}

\author{
Hyosun Kim ${ }^{1}$, Yasushi NAKajima ${ }^{2}$, Hwankyung Sung ${ }^{3}$, Dae-Sik Moon ${ }^{4,5}$, And Bon-Chul Koo ${ }^{1}$ \\ 1 Astronomy Program, Department of Physics and Astronomy, Seoul National University, Seoul 151-742, Korea \\ E-mail: hkim@astro.snu.ac.kr6koo@astrohi.snu.ac.kr \\ 2 National Astronomical Observatory of Japan, 2-21-1 Osawa, Mitaka, Tokyo 181-8858, Japan \\ 3 Department of Astronomy and Space Science, Sejong University, Gunja-dong 98, \\ Gwangjin-gu, Seoul 143-747, Korea \\ E-mail: sungh@sejong.ac.kr \\ ${ }^{4}$ Robert A. Millikan Fellow, Division of Physics, Mathematics, and Astronomy, California Institute of \\ Technology, MC 103-33, Pasadena, CA 91125 \\ E-mail: moon@srl.caltech.edu \\ ${ }^{5}$ Department of Astronomy and Astrophysics, University of Toronto, Toronto, ON M5S 3H4, Canada \\ E-mail: moon@astro.utoronto.ca \\ (Received January 26, 2007; Accepted March 20, 2007)
}

\begin{abstract}
We present wide-field $J H K_{\mathrm{s}}$-band photometric observations of the three compact $\mathrm{H}$ II regions G48.90.3, G49.0-0.3, and G49.2-0.3 in the active star-forming region W51B. The star clusters inside the three compact H II regions show the excess number of stars in the $J-K_{\mathrm{s}}$ histograms compared with reference fields. While the mean color excess ratio $\left(E_{J-H} / E_{H-K_{\mathrm{s}}}\right)$ of the three compact $\mathrm{H}$ II regions are similar to $\sim 2.07$, the visual extinctions toward them are somewhat different: $\sim 17$ mag for G48.9-0.3 and G49.0-0.3; 23 mag for G49.2-0.3. Based on their sizes and brightnesses, we suggest that the age of each compact H II region is $\leq 2$ Myr. The inferred total stellar mass, $\sim 1.4 \times 10^{4} M_{\odot}$, of W51B makes it one of the most active star forming regions in the Galaxy with the star formation efficiency of $\sim 10$ $\%$.
\end{abstract}

Key words : infrared: stars — ISM: H II regions — ISM: individual (W51B) — stars: formation — stars: luminosity function, mass function

\section{INTRODUCTION}

W51B is a giant molecular cloud (GMC) complex located at the tangential point of the Sagittarius spiral arm of the Galaxy, and it is a very active starforming region that consists of several compact H II regions. In radio continuum and molecular line images, the compact $\mathrm{H}$ II regions appear to be still embedded in parental molecular clouds, indicating that they are relatively young (e.g. Bieging 1975; Koo \& Moon 1997; Koo 1997, 1999; Moon \& Park 1998). The H II regions also show strong X-ray emission likely caused by magnetic activities of internal young stars (Koo et al. 2002). The line-of-sight velocities $\left(61-70 \mathrm{~km} \mathrm{~s}^{-1}\right)$ of the sources in W51B are greater than the maximum kinematic velocity $\left(54-58 \mathrm{~km} \mathrm{~s}^{-1}\right)$ permitted by the Galactic rotation (Koo 1999, and references therein). This has been known as the "high-velocity stream" in this region which might have resulted from the spiral density wave associated with the Sagittarius spiral arm. The southern part of W51B appears to show an expanding ring structure with two molecular clumps associated with the compact H II regions G48.9-0.3 and G49.00.3 (Moon \& Park 1998). Using 21-cm H I absorption

Corresponding Author: H. Kim spectra toward the compact $\mathrm{H}$ II regions, Kolpak et al. (2003) determined the distance to W51B to be $\sim 5.6$ kpc.*

One of the most interesting features of W51B is its very high star formation efficiency (SFE): while the Galactic average value is 1-2\% (Myers et al. 1986), Moon \& Koo (1994) obtained $16 \%$ for the overall SFE of W51B and Koo (1999) did $7 \%$ and $15 \%$ for the north-eastern and south-western parts of W51B, respectively. Most of the previous studies of the star formation in W51B, including the ones carried out by Moon \& Koo (1994) and Koo (1999), have exclusively used radio contiuum and molecular line data. Recently, Nanda Kumar et al. (2004) have obtained several smallfield $\left(=90^{\prime \prime}\right)$ near-infrared (NIR) images of W51B, and identified a new, small-scale clumpy structure of star clusters of the compact $\mathrm{H}$ II regions. However, the observations of Nanda Kumar et al. (2004) were somewhat shallow, only reaching $\sim 16.2, \sim 14.4$, and $\sim 15.8$ mag for $J$-, $H$-, and $K$-band, respectively. This rather shallow exposure, together with their small field of view, made it difficult to study fainter, extended stellar populations in W51B. They calculated the mass of

\footnotetext{
${ }^{*}$ We will use $5.6 \mathrm{kpc}$ as the distance to W51B in this paper.
} 


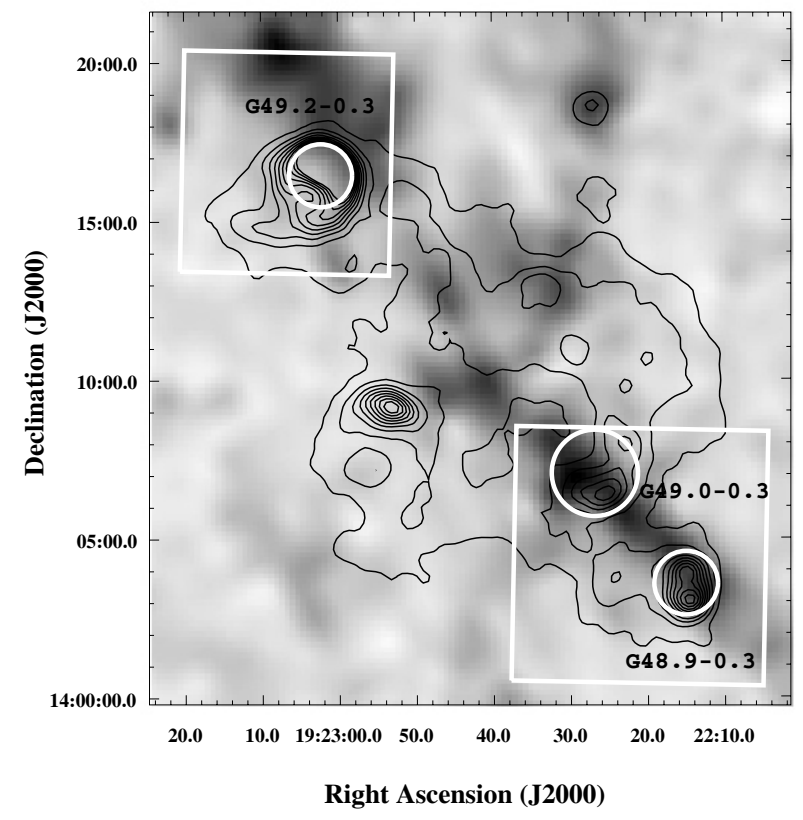

Fig. 1. - Gray-scale map of the ${ }^{13} \mathrm{CO} \mathrm{J}=1-0$ line emission integrated over $v_{\mathrm{LSR}}$ between +45 and $+75 \mathrm{~km} \mathrm{~s}^{-1}$ (Carpenter \& Sanders 1998), superposed on the black contours of the 21-cm radio continuum map (Koo \& Moon 1997). The contour levels are $0.1,0.2,0.3,0.4,0.5,0.6,0.7,0.8$, 0.9 , and $1.0 \mathrm{Jy} \mathrm{beam}^{-1}$. The white boxes and circles represent the NIR observation fields and the NIR (especially $K_{\mathrm{s}}$-band) nebulous area (see Figure 5): $S W$-Field including G48.9-0.3 and G49.0-0.3 (from SW to NE), and NEField including G49.2-0.3. The peak positions of the radio continuum are consistent with those of the NIR nebulosity.

each cluster assuming an average visual extinction of 7 mag. With such moderate extinction, however, all of OB-type members should be visible in second Palomar Observatory Sky Survey (POSS II) red image $\left(R_{c, \lim }\right.$ $=20.8 \mathrm{mag}$, Reid et al. 1991), while no optical counterpart is known. This implies therefore the extinction toward W51B H II regions must be larger than 7 mag. In this paper, we obtained deeper NIR photometric data that cover much more extended regions of W51B. With these new data, we find the initial mass function and the total stellar mass of star clusters in W51B, as well as NIR-based SFE of W51B. Our new observations confirm the results of the previous radio observations that $\mathrm{W} 51 \mathrm{~B}$ is one of the most active star forming regions in the Galaxy.

\section{OBSERVATIONS}

We carried out near-IR $J H K_{\mathrm{s}}$-band photometric observations of W51B using Wide-field InfraRed Camera (WIRC) aboard the 5-m Palomar Hale telescope on 2003 June 17 and 18. WIRC is equipped with a HAWAII-II $\mathrm{HgCdTe} 2 \mathrm{~K}$ array and the pixel scale is $0.25^{\prime \prime}$ pixel $^{-1}$, providing a $8.7^{\prime} \times 8.7^{\prime}$ field of view. The typical seeing was $1^{\prime \prime}$ over the observations. We selected two fields based on the previous radio observations, and Figure 1 shows the locations of the observed fields superimposed on previous radio images. As in Figure 1, one field includes G48.9-0.3 and G49.0-0.3, while the other field contains G49.2-0.3, the brightest radio continuum source in W51B (Koo \& Moon 1997), at its center. We shall call the former $S W$-Field and the latter $N E$-Field hereafter. We obtained 30 and 60 dithered frames of $S W$-Field and NE-Field respectively, for each $J H K_{\mathrm{s}}$ band. The exposure of individual frame was 10 sec. Each dithered frame was shifted and combined after subtracting dark and median-combined sky frames and correcting flat field. Figures 2 and 3 present the final three-color images of the two fields, where we can identify extended emission around the $\mathrm{H}$ II regions due to Br- $\gamma$ emission in the $K_{\mathrm{s}}$ band.

In order to find stars in our WIRC images, we iteratively used the PSF-fitting program in $\mathrm{IRAF}^{\dagger}$ /DAOPHOT until the residuals show no apparent stars left in the images. As a result, we have detected $>11000$ and $\geq 7000$ stars in the $S W$-Field and NE-Field, respectively. Among them $\sim 10000$ and $\sim 6000$ stars have signal-to-noise ratio greater than 10 in all the $J H K_{\mathrm{s}}$ bands, within 10- $\sigma$ limiting magnitudes of $J=19.8$, $H=19.0$, and $K_{\mathrm{s}}=17.9 \mathrm{mag}$. For photometric and astrometric solutions, we used $\sim 200$ stars of 2 MASS Point Source Catalog $\ddagger$ in our fields. The magnitudes of the faintest stars were $J=21.5 \pm 0.5, H=19.7 \pm$ 0.2 , and $K_{\mathrm{s}}=19.2 \pm 0.4$ for $S W$-Field and $J=21.5$ $\pm 0.5, H=19.8 \pm 0.2$, and $K_{\mathrm{s}}=19.5 \pm 0.5$ for $N E$ Field respectively, while the magnitude of the brightest and unsaturated star was $\sim 10$ in all bands. Our astrometric solutions gave $1-\sigma$ uncertainties of $\sim 0^{\prime \prime} .3$ for $S W$-Field and $\sim 0^{\prime \prime} 1$ for $N E$-Field with respect to the 2MASS system.

\section{Star Clusters in W51B}

\section{(a) Membership Selection}

In order to investigate the existence of noticeable star clusters inside the $\mathrm{H}$ II regions, we first examine the stellar number density map as in Figure 4 which was obtained by Gaussian smoothing of number of stars with $15^{\prime \prime}$ FWHM. It shows clusterings of stars inside the three compact H II regions. Although there are many stars at south-east of NE-Field, they should not be regarded as cluster members because they are randomly distributed outside the cloud. They may be background stars behind W51B GMCs. In order to determine the cluster members and examine their properties, we define the cluster regions and the corresponding

IRAF (Imaging Reduction \& Analysis Facility) is distributed by the National Optical Astronomy Observatories, which are operated by the Association of Universities for Research in Astronomy, Inc., under cooperative agreement with the National Science Foundation.

${ }^{\ddagger}$ http://www.ipac.caltech.edu/2mass/ 


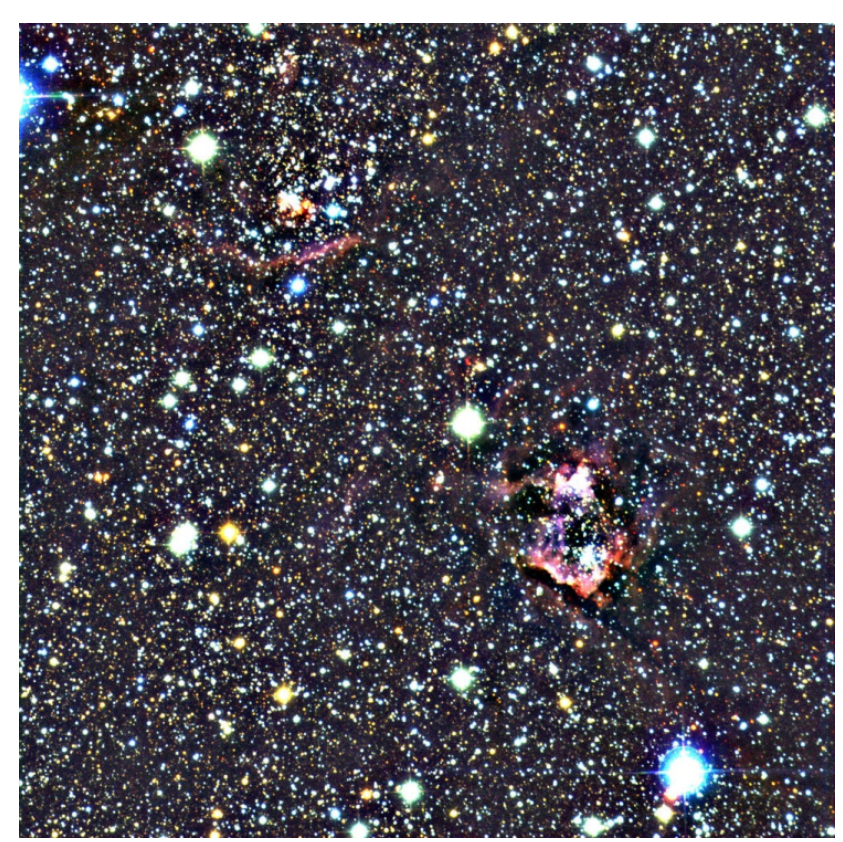

Fig. 2.- $J, H$, and $K_{\mathrm{s}}$ three-color image of $S W$-Field, including the two compact $\mathrm{H}$ II regions G48.9-0.3 and G49.0-0.3. The showing field is $8.2^{\prime} \times 8.2^{\prime}$ centered at $\left(\alpha_{J 2000.0}, \delta_{J 2000.0}\right)=\left(19^{\mathrm{h}} 22^{\mathrm{m}} 21^{\mathrm{s}},+14^{\circ} 4^{\prime} 26^{\prime \prime}\right)$. The $J_{-}, H-$, and $K_{\mathrm{s}}$-band data are represented as blue, green, and red, respectively.

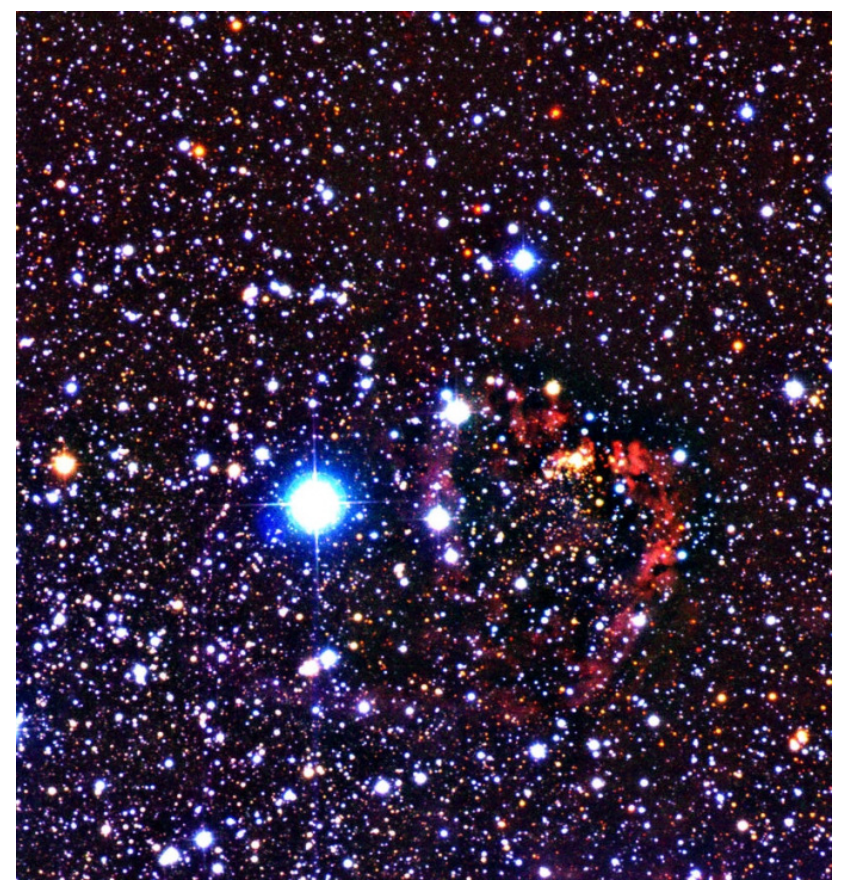

Fig. 3.- Same as Figure 2 but for NE-Field, which includes G49.2-0.3. The showing field is $6.7^{\prime} \times 7.0^{\prime}$ centered at $\left(\alpha_{J 2000.0}, \delta_{J 2000.0}\right)=\left(19^{\mathrm{h}} 23^{\mathrm{m}} 7^{\mathrm{s}},+14^{\circ} 16^{\prime} 47^{\prime \prime}\right)$. reference regions on the basis of the positions of $\mathrm{H}$ II regions, which are described as solid and dashed circles, respectively, in Figure 5. Each reference region is selected just outside the cluster region to minimize the offset effect of spatial density fluctuation. A reference region has the same area with the corresponding cluster region. There are about 600,1500 , and 500 stars in the cluster regions within the H II regions G48.9-0.3, G49.0-0.3, and G49.2-0.3, respectively. These numbers of stars inside the cluster regions are at least 1- $\sigma$ larger than the counts in the randomly selected regions having same area. For G49.2-0.3, we avoid selecting the random regions outside molecular cloud because there must be a lot of background field stars in the off-cloud area.

Figure 6 shows the histograms of $J-K_{\mathrm{s}}$ color of the stars detected at all bands in the direction of three $\mathrm{H}$ II regions. The first bump at $J-K_{\mathrm{s}} \sim 1.4$ is found on the histograms for both cluster and reference regions, thus it should be caused by foreground field stars. With an assumption of $A_{V} \sim 1 \mathrm{mag} \mathrm{kpc}^{-1}$, they seem to be mainly composed of the Sagittarius arm which extends through a range of $\sim 3-6 \mathrm{kpc}$ at $l \simeq 49^{\circ}$ (Wainscoat et al. 1992). We see outstanding bumps toward G48.9-0.3 and G49.0-0.3 at $2.2<J-K_{\mathrm{s}}<3.2$, compared to the histograms of the reference regions. In the histogram for G49.2-0.3, the extra bump is at $2.6<J-K_{\mathrm{s}}<4.5$. The inset panel represents the excess number of stars compared to reference region. The excess should be caused by the probable cluster members.

Although the bump at $J-K_{\mathrm{s}} \geq 2.2$ probably includes most of the cluster members (we call them "B" (Bump) members hereafter), we may fail to select the pre-main-sequence (PMS) stars which are generally redder than the main-sequence (MS) stars due to the intrinsic emission from the circumstellar dust. Thus, we need to separately include the PMS stars into the cluster member candidates. We use $H-K_{\mathrm{s}}$ vs. $J-H$ color-color diagram (CCD) to select PMS stars with intrinsic NIR color excess. In order to distinguish the intrinsic color excess from the reddening by interstellar extinction, we first compute the color excess ratio $E_{J-H} / E_{H-K_{\mathrm{s}}}$ toward W51B in the 2MASS system by a least-squares fit with $3-\sigma$ clipping. Figure 7 shows the CCD of bright stars $\left(K_{\mathrm{s}}<13\right)$ with each color error less than 0.01 mag. The stars after $3-\sigma$ clipping yield the ratios of $2.08 \pm 0.04$ for $S W$-Field and $2.07 \pm 0.02$ for NE-Field. Those are similar but slightly larger than 1.98 of Fitzpatrick (1999) provided from extensive data in the Galaxy or $1.97 \pm 0.01$ of Han (2001) obtained for a $25^{\prime} \times 25^{\prime}$ field including whole W51B giant molecular cloud region and large off-cloud region. Because our fields are focused on the cloud core regions, there could be a little difference from the value calculated for a wide field. Hereafter, we apply the color excess ratio of 2.07 in the direction of the $\mathrm{H}$ II regions in W51B and use the relations of Fitzpatrick in order to estimate total extinction. 

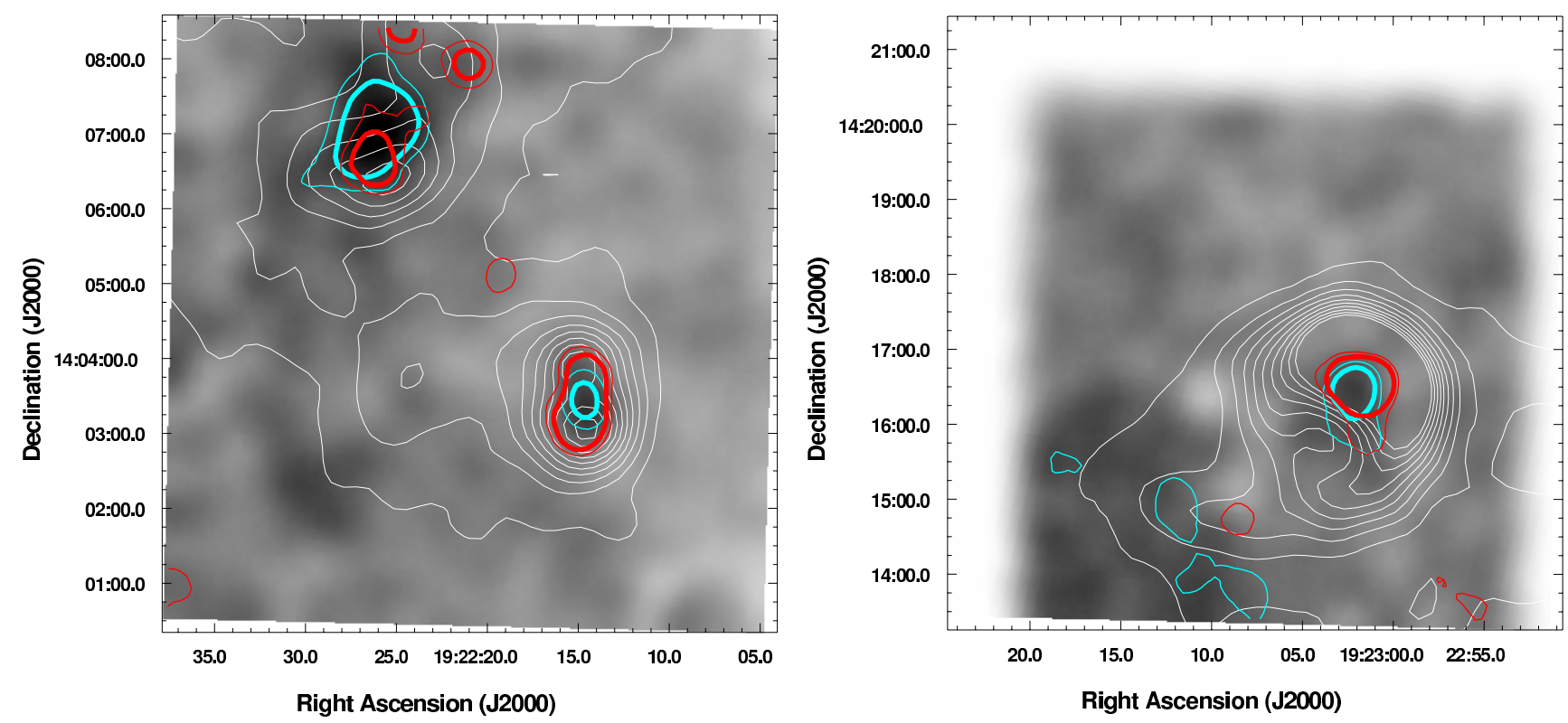

Fig. 4. - Gray-scale map of the number density of stars detected in all bands, superposed on red and blue contours of the number densities of "P" and "B" members, respectively (see text), for each field. The colored thick and thin contours represent 3- $\sigma$ and 2- $\sigma$ of the number density where $\sigma$ is the standard deviation of each kind of density. The white contours are the 21-cm radio continuum whose levels are given in Figure 1. The left and right panels are for $S W$-Field and NE-Field, respectively.
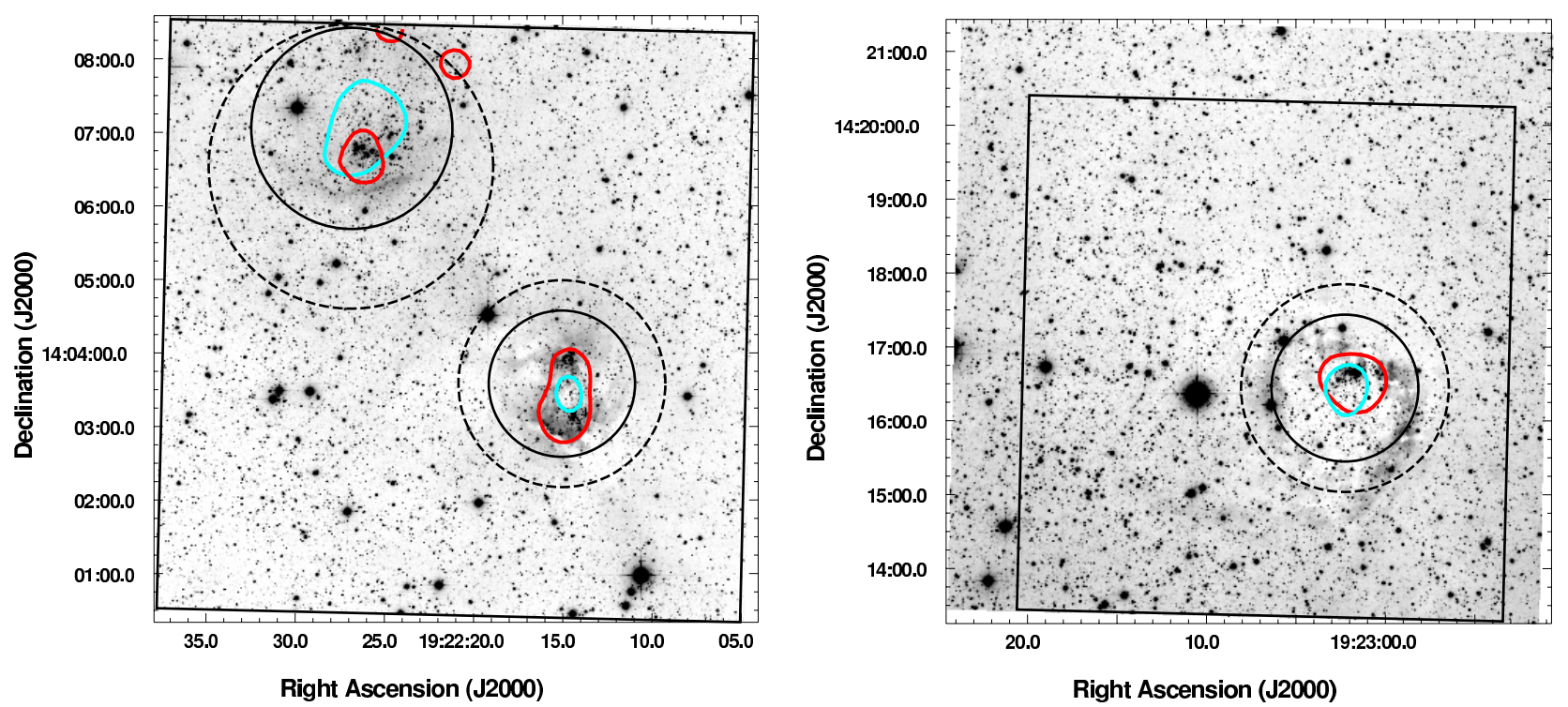

Fig. 5.- $K_{\mathrm{s}}$-band images for $S W$-Field (left) and $N E$-Field (right). For $N E$-Field, the observed $J$-, $H$-, and $K_{\mathrm{s}}-$ band frames were shifted each other, and we use only the matched area which is inside the black box. The solid and dashed circles represent the cluster and reference regions, respectively. The reference region has the same area with the corresponding cluster region. Also shown are the cluster-core regions which are defined inside the 3- $\sigma$ stellar density contours in Figure 4 . 


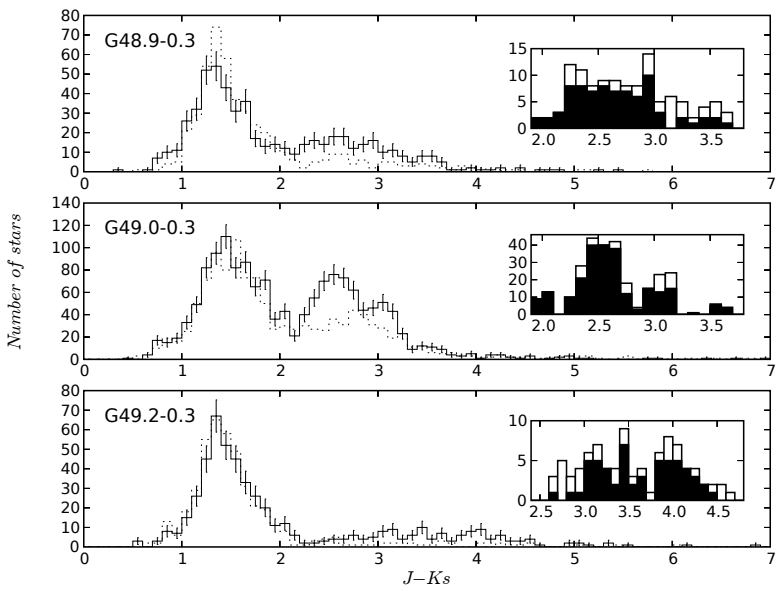

Fig. 6. - Histograms along $J-K_{\mathrm{s}}$ color. Solid and dotted lines show the histograms for a cluster region and the corresponding reference region, respectively. The error bar denotes the Poisson noise level in each bin. The difference for the second bump in the number of stars between the cluster region and the reference region is displayed as an inset of each panel. The white and black histograms represent the numbers of PMS and the others, respectively.
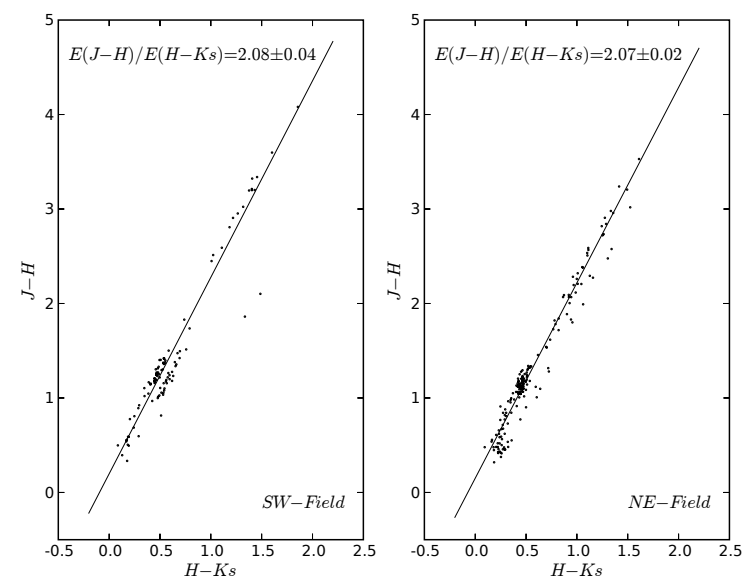

Fig. 7. - Color-color diagram of the bright stars $\left(K_{\mathrm{S}}<13\right)$ in each field with good photometry $\left(H-K_{\mathrm{s}}\right.$ and $J-H$ color errors less than $0.01 \mathrm{mag}$ ). Reddening slopes are obtained using a least-squares fit with $3-\sigma$ clipping. The left panel is for $S W$-Field, and the right one for NE-Field. The line in each panel shows the fitted reddening slope, both of which are $\sim 2.07$.
Figure 8 is $H-K_{\mathrm{s}}$ vs. $J-H$ CCDs where the reddening slope of the dashed lines is determined by the color excess ratio obtained in the previous paragraph. The area between the two dashed lines is the "reddening zone" in which the reddened dwarfs and giants fall. Stars with intrinsic infrared excess emission can fall into the right of the reddening zone. Lada \& Adams (1992) called this region as "excess zone". The sources in the excess zone are generally pre- and post-main-sequence stars with circumstellar dust and galaxies with redshifts $\mathrm{z}>0.1$. Toward the compact H II regions, most of them should be pre-main-sequence stars. We mark these stars in red color, except possible spreading foreground stars with $J-K_{\mathrm{s}}<2.2$. We call them "P" (PMS) members hereafter. And we mark the "B" members in blue color. Most of the "B" members are MS stars, though some of them are marked in both colors which indicate that they are probably PMS stars. Since most stars in these categories are located above the reddening line of $3 M_{\odot}$ stars in $J-K_{\mathrm{s}}$ vs. $J$ color-magnitude diagrams in Figure 9, we suppose the observed cluster members to be OB-type MS stars and/or intermediate- to highmass PMS stars. We note the numbers of stars in the two categories in Table 1. The selected cluster member candidates are 168, 589, and 120 for G48.9-0.3, G49.00.3 , and G49.2-0.3, respectively. These candidates, however, include some field star contamination though we already excluded most of the foreground field stars composing the bump at $J-K_{\mathrm{s}}<2.2$ in Figure 6 .

In order to analyze how many field stars are contained, we choose out the stars inside the reference region in each category using the same method, and compare the number of stars between cluster and reference regions. We confirm that at least $60-80 \%$ of the selected member candidates are statistically reliable for G48.9-0.3 and G49.2-0.3, while the reliability is $40 \%$ for G49.0-0.3. Since G49.0-0.3 is sparsely distributed and the extinction varies a lot, the cluster members could not be determined more reliably. However, most of the field star contamination contributes to the low luminosity regime $(J>18 \mathrm{mag})$, thus their contribution to the total luminosity is expected to be insignificant.

\section{(b) Interstellar Extinction toward W51B Clus- ters}

We expect that the average interstellar extinction toward the cluster can be simply obtained from the mean photometric color of members. Since the range of the intrinsic NIR colors of OB-type MS stars is very narrow $\left(\Delta\left(J-K_{\mathrm{s}}\right)_{\text {intrinsic }} \sim 0.2 \mathrm{mag}\right)$ compared to the $J-K_{\mathrm{s}}$ range of sources in the observed field, the $J-K_{\mathrm{s}}$ color can be a good indicator of extinction.

We transform the observed $J-K_{\mathrm{s}}$ colors of MS member candidates to the visual extinctions using

$$
A_{V}=6.31\left[\left(J-K_{\mathrm{s}}\right)_{\mathrm{obs} .}-\left(J-K_{\mathrm{s}}\right)_{\text {intrinsic }}\right],
$$

which is obtained from Fitzpatrick's relations converted 
TABLE 1

Number of Cluster Member Candidates

\begin{tabular}{cccc}
\hline \hline H II region & "B" members & "P" members & "B" \& "P" members \\
\hline G48.9-0.3 & 143 & 66 & 41 \\
G49.0-0.3 & 560 & 105 & 76 \\
G49.2-0.3 & 109 & 45 & 34 \\
\hline
\end{tabular}

Note. - The fourth column represents the number of stars in both categories, and we include these stars into PMS member candidates. MS member candidates are considered as the stars in "B" but not "P" category.

to the 2MASS system ${ }^{\S}$ which is based on Carpenter (2001). We assume the intrinsic $J-K_{\mathrm{s}}$ color of OB stars to be approximately zero. PMS stars must possess redder intrinsic colors though there is not a definite model explaining the color of massive PMS stars yet. We assume the average extinction of PMS stars is identical to that of MS stars. The mean visual extinctions for the member candidates are $17 \mathrm{mag}$ for both G48.9-0.3 and G49.0-0.3, and 23 mag for G49.20.3. Similarly, mean $J$-band extinctions are obtained from $A_{J}=1.73\left(J-K_{\mathrm{s}}\right)_{\mathrm{obs}}$ : $4.6 \mathrm{mag}$ for G48.9-0.3 and G49.0-0.3, and $6.2 \mathrm{mag}$ for G49.2-0.3. The photometric diagrams of G49.2-0.3 show a wide separation between the cluster and foreground stars at $J-K_{\mathrm{s}} \sim$ 2 , which is consistent with the higher extinction value than the other two clusters.

\section{(c) Spatial Distribution of Member Stars}

In order to see the clustering feature of the member candidates rigorously, we describe the number densities of the "P" and "B" members as red and blue contours in Figure 4, overlapped on the gray-scale map for the number density of all observed stars. We define the cluster-core region inside the contour of 3- $\sigma$ where $\sigma$ is the standard deviation of the density fluctuations. In Table 2, we display the stellar column and volume densities of each group inside each cluster-core region after statistical subtraction of the stars outside the region under consideration. They are the lower limits of the cluster densities because the lowest mass of the marginally detectable members are $2-5 M_{\odot}$ corresponding to the $10-\sigma$ limiting magnitudes.

In G48.9-0.3, both MS and PMS members are well confined inside the area of radio continuum, elongated from north to south. The PMS members appear to be peanut-shaped. G49.0-0.3 shows a large and bright density peak of "B" members. The PMS stars are con-

\footnotetext{
$\S$ http://www.ipac.caltech.edu/2mass/
}

centrated on the southern part of it near the radio continuum peak. There are two small PMS clusters at rather distant places $\left(\sim 2^{\prime}\right)$ on the north-west of G49.0 0.3 . The central region of G49.2-0.3 is crowded inside a small place, while the radio continuum source is much larger than the previously mentioned two other H II regions. If G49.2-0.3 is a "blister-type" H II region being suggested from the radio continuum morphology and the distribution of molecular gas (e.g. Koo 1999), the large size of the ionized gas could be explained by low density of ambient medium.

\section{STAR FORMATION EFFICIENCY}

With the $J$-band magnitudes of member stars, we find the initial mass function (IMF) and the total stellar mass of each cluster. We use $J$-band luminosity which is less affected by the intrinsic excess emission of PMS stars than $H$ - or $K_{\mathrm{s}}$-band luminosity. We transform the observed $J$ magnitudes of all member candidates to the absolute magnitudes applying the mean extinction of the member candidates derived at $\S$ III (b). The transformation from the absolute $J$-magnitude to the initial mass is accomplished using the theoretical massluminosity relation from the Geneva models and theoretical colors of MS stars (Schaller et al. 1992; Bessell et al. 1998) and Testi et al. (1998) for PMS stars less massive than $3 M_{\odot}$. Since massive PMS stars $\left(>3 M_{\odot}\right)$ would be located very close to the main sequence in the mass-luminosity relation, the MS relation is applied for them. We assume the age of clusters to be $1 \mathrm{Myr}$, and the derived total mass is not sensitive to the age. Figure 10 shows the resultant IMF for each cluster. The number of stars increases as the stellar mass decreases at the high mass regime, while the detection limit causes the IMFs to decrease at the lower mass regime. For comparison, we plot the IMF of the young open cluster NGC 2264 (Sung et al. 2004, solid curve) extrapolated using the IMF of the solar neighborhoods (Lee \& Sung $1995)$ for the massive stars $\left(>30 M_{\odot}\right)$ and vertically 
TABLE 2

Number of Stars in Each Cluster-Core Region

\begin{tabular}{cccrrc}
\hline \hline H II region & Name $^{\mathrm{a}}$ & Number & Area $\left(\square^{\prime \prime}\right)$ & $N / A_{\mathrm{pc}^{2}}{ }^{\mathrm{b}}$ & $N / V_{\mathrm{pc}^{3}}{ }^{\mathrm{c}}$ \\
\hline \multirow{2}{*}{ G48.9-0.3 } & P4855044-0017085 & $36 \pm 7$ & 2450 & $63 \pm 12$ & $20 \pm 4$ \\
& B4855164-0016594 & $23 \pm 6$ & 449 & $218 \pm 55$ & $160 \pm 40$ \\
\hline G49.0-0.3 & P4859241-0018016 & $8 \pm 4$ & 1125 & $30 \pm 16$ & $14 \pm 7$ \\
& B4859462-0017400 & $122 \pm 16$ & 3674 & $142 \pm 18$ & $36 \pm 5$ \\
\hline G49.2-0.3 & P4912136-0020508 & $16 \pm 5$ & 1989 & $34 \pm 10$ & $12 \pm 4$ \\
& B4912111-0021025 & $26 \pm 7$ & 1064 & $105 \pm 27$ & $50 \pm 13$ \\
\hline
\end{tabular}

a The position of the local peak in Galactic coordinates were used to name the clustering groups. The first letter represents "PMS" and "Bump", respectively.

b Column density. $N / A_{\mathrm{pc}^{2}}=4262 N / A_{\square}$ " adopting the distance of $5.6 \mathrm{kpc}$.

c Volume density. $N / V_{\mathrm{pc}^{3}}=66430 N / A_{\square^{\prime \prime}}^{3 / 2}$ if we assume the cluster is spherical.

shifted to fit our data within $1.0 \leq \log \left(M / M_{\odot}\right) \leq 1.3$. Sung et al. noticed that NGC 2264 has similar shape of IMF to the Pleiades and the Trapezium. The IMFs of G48.9-0.3 and G49.0-0.3 agree well with the comparison IMF at $M>5-6 M_{\odot}$. For G49.2-0.3, the consistent range is $M>10 M_{\odot}$ due to high extinction toward this cluster.

In order to estimate total mass of a cluster, the universality of IMF is assumed. The total mass is determined from

$$
\mathrm{M}_{\mathrm{tot}}=\int \mathrm{M} \xi(\log \mathrm{M}) \mathrm{d} \log \mathrm{M}
$$

where $\xi$ is the IMF of NGC 2264 which described in the previous paragraph. $\mathrm{T}$ he upper limit of the integral is decided on the mass of the most massive stars, and the lower limit is fixed to $0.01 M_{\odot}$. Table 3 represents the total mass of the cluster associated with each H II region. We also list the stellar mass of the only observed stars, instead assuming the universality of IMF. Undetected stars would contribute to the cluster mass in amount of at least twice than the observed massive stars do.

We calculate the star formation efficiency (SFE) which is defined as the ratio of the stellar mass to the total mass of stars plus cloud, using total stellar mass in Table 3 and the molecular cloud mass in Table 1 of Koo (1999). The overall SFEs are $7 \%$ for the cloud SW including G48.9-0.3 and G49.0-0.3, and 17 $\%$ for the cloud NE including G49.2-0.3. Even if we only use the stellar mass of the observed massive stars, the efficiencies are estimated to be $3-4 \%$. Because no cluster is composed of only massive OB stars, the SFEs of W51B appear to be clearly high compared to the Galactic average value of $1-2 \%$. Besides, the high
SFEs are lower limits because we ignore the possible smaller clusters/associations along the GMCs. For explanations about the high SFE, a cloud-cloud collision between high-velocity $\left(68 \mathrm{~km} \mathrm{~s}^{-1}\right)$ cloud and the W51 GMC (Carpenter \& Sanders 1998) and the enhancement due to the spiral density wave (Koo 1999; Nanda Kumar et al. 2004) have been speculated.

Table 2 of Lada \& Lada (2003) shows that the SFEs for nearby embedded clusters range from $\sim 10 \%$ to $30 \%$ when the cloud core masses are adopted, not the masses for entire giant molecular clouds. The compressed core masses presented in that paper are less than $1000 M_{\odot}$, which is at least 10 times less than the entire mass of W51B GMCs. We cannot compare the core SFEs to the overall SFEs of W51B GMCs until the individual core masses are obtained by high resolution observations.

\section{AGE OF CLUSTERS}

As the stars emit Lyman continuum photons, the $\mathrm{H}$ II region increases in size. Therefore, in a given size of the H II region, the age is inversely proportional to the Lyman continuum photon flux. If the $\mathrm{H}$ II region expands in a uniform medium, the radius of an $\mathrm{H}$ II region as a function of time is given by

$$
r(t)=r_{i}\left(1+\frac{7 c_{i} t}{4 r_{i}}\right)^{4 / 7}
$$

where

$$
r_{i}=\left(\frac{3 N_{C}^{\prime}}{4 \pi n_{e} n_{H} \beta}\right)^{1 / 3}
$$

is the initial Strömgren radius, $c_{i}$ is the sound speed in the ionized gas $\left(\sim 10 \mathrm{~km} \mathrm{~s}^{-1}\right.$ for $\left.10^{4} \mathrm{~K}\right), n_{e}$ and 
TABLE 3

Total Stellar Mass Estimations

\begin{tabular}{ccrc}
\hline \hline H II region & Method & $\begin{array}{c}\text { Mass Range } \\
\left(M_{\odot}\right)\end{array}$ & $\begin{array}{r}\text { Total Stellar Mass } \\
\left(M_{\odot}\right)\end{array}$ \\
\hline \multirow{2}{*}{ G48.9-0.3 } & 1 & $0.01 \sim 20$ & 2400 \\
& 2 & $1.3 \sim 20$ & 800 \\
\hline G49.0-0.3 & 1 & $0.01 \sim 25$ & 4200 \\
& 2 & $0.8 \sim 25$ & 2300 \\
\hline G49.2-0.3 & 1 & 0.01 & $\sim 100$ \\
& 2 & 2.5 & $\sim 100$ \\
& & & 1300 \\
\hline
\end{tabular}

Note. - For the method (1), we assume the combined IMF of NGC 2264 and solar neighborhoods as a universal IMF in order to estimate total mass including undetected low-mass stars. Method (2) is the stellar mass of the observed stars without the assumption.
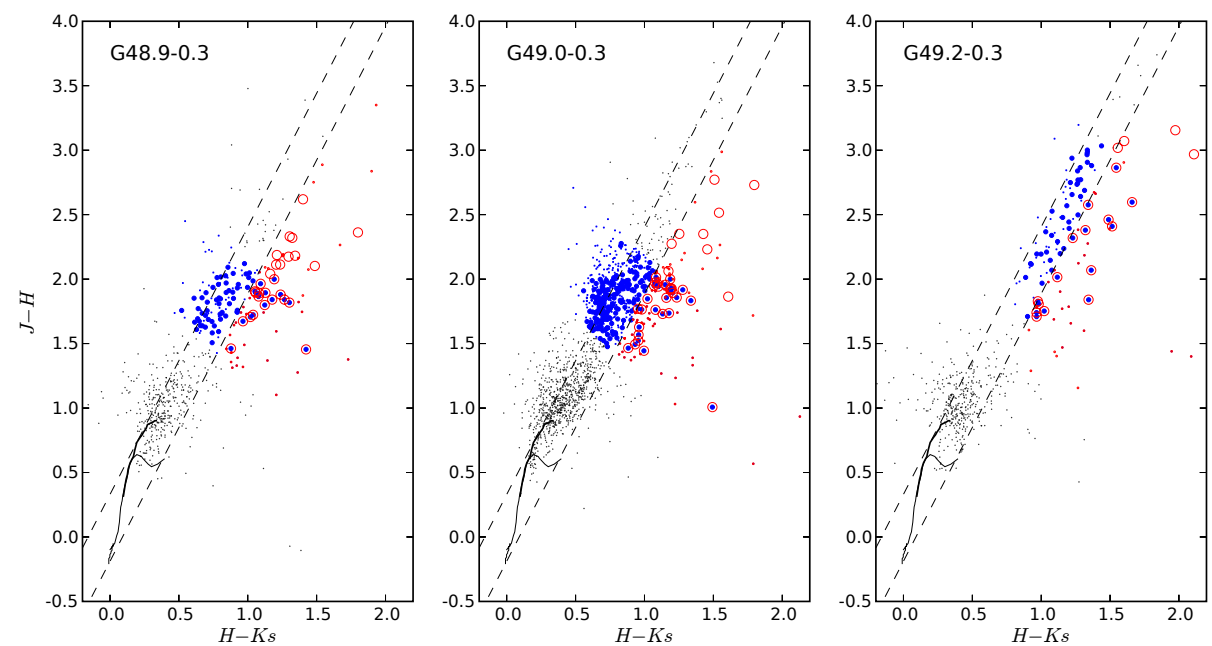

Fig. 8. - Color-color diagram of stars in each cluster region. The member candidates are marked in red and blue colors. The red marks are "P" members, while the blue marks are the "B" members. The red open circles and the blue closed circles represent the member candidates with small color error $(<0.1 \mathrm{mag})$, and the colored dots are the candidates with larger error. The thin and thick solid curves are the loci occupied by the unreddened dwarfs and giants (Bessell \& Brett 1988; Koornneef 1983). The dashed lines are parallel to the reddening vector. 

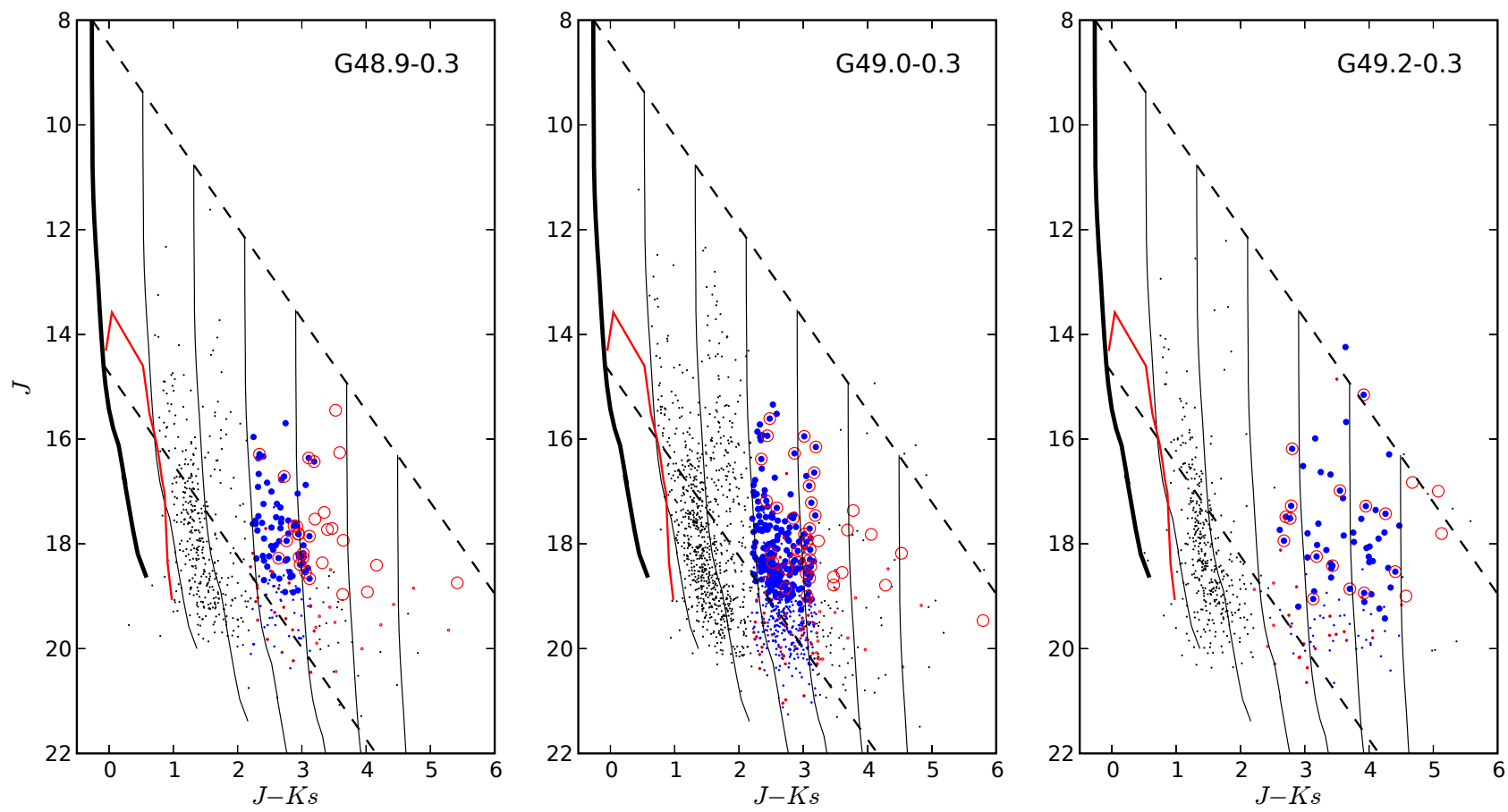

Fig. 9.- Color-magnitude diagram of stars in each cluster region. The black, thick curve represents the unreddened zero-age main-sequence (ZAMS) relation for $\mathrm{Z}=0.020$ from the Geneva models (Schaller et al. 1992; Bessell et al. 1998), adopting the distance of $5.6 \mathrm{kpc}$ to the compact H II regions. The dashed lines are parallel to the reddening vector of ZAMS star having mass of $120 M_{\odot}$ (up) and $3 M_{\odot}$ (down). The black, thin curves are reddened ZAMS corresponding with $A_{V}$ of 5 , 10, 15, 20, 25, and $30 \mathrm{mag}$. Additionally, the red curve is the loci of unreddened PMS stars with age of $1 \mathrm{Myr}$ (Testi et al. 1998). The red and blue marks represent the member candidates which are described in Figure 8.
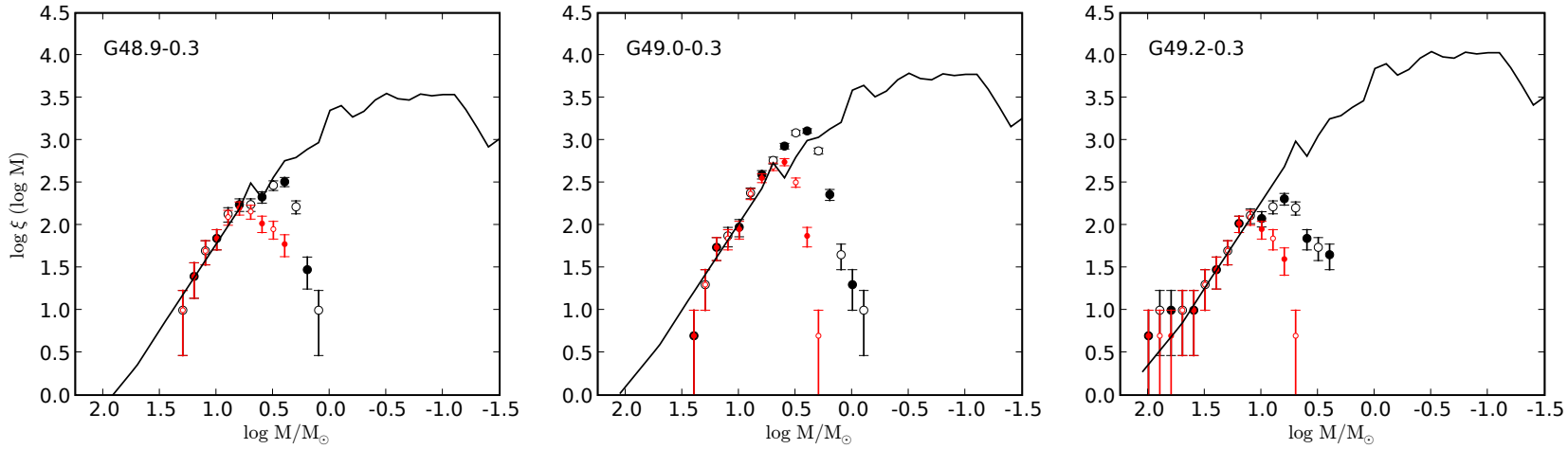

Fig. 10. - Initial mass functions for all cluster member candidates (black) and the member candidates with color error less than $0.1 \mathrm{mag}$ (red). We count the stars within the mass interval $\Delta \log \left(M / M_{\odot}\right)=0.2$ and in the same-sized bin shifted by 0.1 to reduce the binning effect. They are represented as open and closed circles. The error bars denote the statistical fluctuations in the counts under the assumption that the Poisson statistics governs the fluctuations. The initial mass function of NGC 2264 (solid curve) is vertically shifted to fit our data and used to estimate total mass of W51B. 
TABLE 4

Properties of the Clusters Associated with Three H II Regions

\begin{tabular}{cccccc}
\hline \hline H II region & $\begin{array}{c}\left\langle A_{V}\right\rangle \\
(\mathrm{mag})\end{array}$ & $\begin{array}{c}\text { Radius } \\
(\mathrm{pc})\end{array}$ & $\begin{array}{c}\text { Spectral Type of } \\
\text { the most massive star }\end{array}$ & $\begin{array}{c}\log N_{C}{ }^{\mathrm{a}} \\
\left(\mathrm{s}^{-1}\right)\end{array}$ & $\begin{array}{c}\text { Age } \\
(\mathrm{Myr})\end{array}$ \\
\hline G48.9-0.3 & 17 & 1.6 & B0 & $47.85(46.90)$ & $0.6(1.1)$ \\
G49.0-0.3 & 17 & 2.2 & O9 & $48.31(48.23)$ & $0.8(0.9)$ \\
G49.2-0.3 & 23 & 2.7 & O4 & $50.43(50.25)$ & $0.3(0.3)$ \\
\hline
\end{tabular}

a Lyman continuum photon flux of all members (and the members without NIR excess).

b Dynamic age of the H II region using all members (and the members without NIR excess).

$n_{H}$ are the ambient densities of electron and atomic hydrogen, $\beta$ is the recombination coefficient $(\sim 3 \times$ $10^{-13} \mathrm{~cm}^{3} \mathrm{~s}^{-1}$ for $\left.T_{e}=10^{4} \mathrm{~K}\right)$, and $N_{C}^{\prime}$ is the effective Lyman continuum photon flux (e.g. Dyson \& Williams 1980). $N_{C}^{\prime}$ is evaluated assuming $90 \%$ of the Lyman photons are absorbed by dust within the $\mathrm{H}$ II region (Wood \& Churchwell 1989). If we adopt $n_{e}=n_{H}$, the age of an $\mathrm{H}$ II region is simplified as follows:

$$
\begin{aligned}
t \simeq & 0.14 \operatorname{Myr}\left(\frac{\mathrm{n}_{\mathrm{e}}}{10^{3} \mathrm{~cm}^{-3}}\right)^{1 / 2}\left(\frac{\mathrm{r}}{1 \mathrm{pc}}\right)^{7 / 4}\left(\frac{\mathrm{N}_{\mathrm{C}}^{\prime}}{10^{48} \mathrm{~s}^{-1}}\right)^{-1 / 4} \\
& -0.017 \operatorname{Myr}\left(\frac{\mathrm{n}_{\mathrm{e}}}{10^{3} \mathrm{~cm}^{-3}}\right)^{-2 / 3}\left(\frac{\mathrm{N}_{\mathrm{C}}^{\prime}}{10^{48} \mathrm{~s}^{-1}}\right)^{1 / 3}
\end{aligned}
$$

Although the three H II regions in W51B are not spherical, we may roughly estimate the age of the H II regions from equation (5). The mean electron density of W51B H II regions is $\simeq 10^{3} \mathrm{~cm}^{-3}$ (Mufson \& Liszt 1979; Mehringer 1994). The present sizes of the H II regions are decided from the appearance of the $21-\mathrm{cm}$ radio continuum emission $\left(0.3 \mathrm{Jy} \mathrm{beam}^{-1}\right.$ contour in Figure 1), and are presented in Table 4. In the table, we also present the spectral type of the most massive star of a cluster and the total Lyman continuum photon flux that the cluster members emit at ZAMS stage (Panagia 1973), together with the resultant age. The Lyman flux and the inferred age using only the stars which are expected to be already in main-sequence are put in parentheses. We derive the age to be the order of or younger than $1 \mathrm{Myr}$ for all H II regions. However, even in an ideal case, the dynamic age of the H II region may give only the age of the most massive stars of the cluster. In addition, the dynamic age is quite sensitive to the size of $\mathrm{H}$ II region, which depends on the distance. Nevertheless, the deduced dynamic age is roughly consistent with the age estimation via $J H K_{\mathrm{s}}$ excess fraction, which is shown in next paragraph.

The fraction of the stars possessing NIR excess emission is revealed to be another indicator of age (Haisch et al. 2001a, and therein). For a very young embedded cluster NGC 2024 ( $0.3 \mathrm{Myr}$, Meyer 1996), the $J H K$ excess fraction of $58 \% \pm 7 \%$ is derived by Haisch et al. (2000). The fraction decreases as the age increases: $50 \% \pm 7 \%$ (Lada et al. 2000) and $21 \% \pm 5 \%$ (Haisch et al. 2001b) for the Trapezium with age of $\sim 1.5 \mathrm{Myr}$ and IC 348 with $\sim 2.3$ Myr (Palla \& Stahler 2000), respectively. From this tendency, we can use the fractions of PMS member candidates in order to conjecture the age of the W51B clusters. The fractions are 66/168 $(39 \%), 105 / 589(18 \%)$, and $45 / 120$ (38\%) for G48.9 0.3 , G49.0-0.3, and G49.2-0.3, respectively. Subtracting the field star contaminations, the fractions are given by $44 / 108$ (41\%), 39/246 (16\%), 33/89 (37\%) in order. These fractions of $20-40 \%$ imply that the age of the clusters are approximately 2 Myr. It should be the upper limit of the cluster age because we detect only massive OB stars whose NIR excess fraction is expected to be lower than the low-mass stars due to more rapid disk dispersal times (Lada et al. 2000; Haisch et al. 2001b). However, $L$-band observation is needed for robust estimation of cluster age because $K$-band involves risk under the influence of nebulous environments (Haisch et al. 2000).

\section{SUMMARY}

We have presented results from deep $J_{-}, H$-, and $K_{\mathrm{s}}$-band observations of two fields including three compact H II regions along W51B GMC. We set constraints to select the possible cluster members associated with each $\mathrm{H}$ II region. We investigate the IMF using $J$-band luminosity and estimate total stellar mass from a welldefined comparison IMF through the whole mass range (from $0.01 M_{\odot}$ to the mass of the most massive stars). In particular, we find the followings:

- We compute the reddening slope in the direction of W51B in the 2MASS system, by a least-squares fit with $3-\sigma$ clipping. The resultant color excess ratio is $E_{J-H} / E_{H-K_{\mathrm{s}}} \sim 2.07$.

- We define the cluster member candidates as the sources within the distinct bump in the histogram 
along $J-K_{\mathrm{s}}$ color and the sources showing NIR color excess emission in the color-color diagram.

- The mean visual extinctions for the cluster member candidates are $17 \mathrm{mag}$ for both G48.9-0.3 and G49.0-0.3, and 23 mag for G49.2-0.3.

- Each IMF based on $J$-band luminosity corresponds to the IMF of a young open cluster NGC 2264. A universal IMF assumption provides total stellar mass of $\sim 1.4 \times 10^{4} M_{\odot}$, which is nearly 3 times of the observed stellar mass. The overall SFEs are $7 \%$ for the cloud including G48.9-0.3 and G49.0-0.3, and $17 \%$ for G49.2-0.3, which are much higher than the Galactic average value of a few percent.

- We estimate the dynamic age of each H II region to be the order of or younger than $1 \mathrm{Myr}$, in terms of the expansion of Strömgren sphere of a compact $\mathrm{H}$ II region. The $J H K_{\mathrm{s}}$ excess fractions of $20-40 \%$ imply the cluster age of $\sim 2 \mathrm{Myr}$, which should be the upper limit of age in consideration of more rapid disk dispersal times for massive stars.

\section{ACKNOWLEDGEMENTS}

This work was supported by the Korea Science and Engineering Foundation Grant R14-2002-010000 . H. Kim has been partially supported by the BK21 project of the Korean Government. H. Sung acknowledges the support of the Korea Science and Engineering Foundation (KOSEF) to the Astrophysical Research Center for the Structure and Evolution of the Cosmos (ARCSEC) at Sejong University. Finally, we are grateful to Jae-Joon Lee for the technical assistance.

\section{REFERENCES}

Bessell, M. S. \& Brett, J. M., 1988, JHKLM Photometry: Standard Systems, Passbands, and Intrinsic Colors, PASP, 100, 1134

Bessell, M. S., Castelli, F., \& Plez, B., 1998, Model Atmospheres Broad-Band Colors, Bolometric Corrections, and Temperature Calibrations for O-M Stars, A\&A, 333, 231

Bieging, J., 1975, in Lecture Notes in Physics, 42, H II Regions and Related Topics, ed. T. L. Wilson \& D. Downes (Berlin: Springer), 443

Carpenter, J. M., 2001, Color Transformations for the 2MASS Second Incremental Data Release, AJ, 121, 2851

Carpenter, J. M. \& Sanders, D. B., 1998, The W51 Giant Molecular Cloud, AJ, 116, 1856

Dyson, J. E., \& Williams, D. A. 1980, Physics of the Interstellar Medium (New York: Wiley)

Fitzpatrick, E. L., 1999, Correcting for the Effects of Interstellar Extinction, PASP, 111, 63
Haisch, K. E., Lada, E. A., \& Lada, C. J., 2000, A Near-Infrared $L$-Band Survey of the Young Embedded Cluster NGC 2024, AJ, 120, 1396

Haisch, K. E., Lada, E. A., \& Lada, C. J., 2001a, Disk Frequencies and Lifetimes in Young Clusters, ApJ, 553, L153

Haisch, K. E., Lada, E. A., \& Lada, C. J., 2001b, Circumstellar Disks in the IC 348 Cluster, AJ, 121, 2065

Han, S. W., 2001, A Near-InfraRed Photometric Study of the W51B Complex, Master's thesis, Seoul National Univ.

Kolpak, M. A., Jackson, J. M., Bania, T. M., Clemens, D. P., \& Dickey, J. M., 2003, Resolving the Kinematic Distance Ambiguity toward Galactic H II Regions, ApJ, 582, 756

Koo, B. -C., 1997, H I 21 Centimeter Absorption Line Study of the W51 Complex, ApJS, 108, 489

Koo, B. -C., 1999, CO Observations of the W51B H II Region Complex, ApJ, 518, 760

Koo, B. -C., Lee, J. -J., \& Seward, F. D., 2002, An ASCA Study of the W51 Complex, AJ, 123, 1629

Koo, B. -C., \& Moon, D. -S., 1997, Interaction between the W51C Supernova Remnant and a Molecular Cloud. I. H I 21 Centimeter Line Observations, ApJ, 475, 194

Koornneef, J., 1983, Near-infrared photometry. II: Intrinsic Colours and the Absolute Calibration from One to Five Micron, A\&A, 128, 84

Lada, C. J. \& Adams, F. C., 1992, Interpreting Infrared Color-Color Diagrams: Circumstellar Disks around Low- and Intermediate-Mass Young Stellar Objects, ApJ, 393, 278

Lada, C. J. \& Lada, E. A., 2003, Embedded Clusters in Molecular Clouds, ARA\&A, 41, 57

Lada, C. J., Muench, A. A., Haisch, K. E., Lada, E. A., Alves, J. F., Tollestrup, E. V., \& Willner, S. P., 2000, Infrared $L$-Band Observations of the Trapezium Cluster: A Census of Circumstellar Disks and Candidate Protostars, AJ, 120, 3162

Lee, S.-W. \& Sung, H., 1995, The Luminosity Function and Initial Mass Function for the Pleiades Cluster, Journal of Korean Astronomical Society, 28, 45

Mehringer, D. M., 1994, Radio Continuum and Radio Recombination Line Observations of W51, ApJS, 91, 713

Meyer, M. R., 1996, Stellar Populations of Deeply Embedded Young Clusters: NearInfrared Spectroscopy and Emergent Mass Distributions, Ph.D. Thesis, Univ. Massachusetts

Moon, D.-S. \& Koo, B.-C., 1994, Thermal and NonThermal Radio Continuum Sources in the W51 Complex, Journal of Korean Astronomical Society, 27,81 
Moon, D. -S. \& Park, Y. -S., 1998, The High-Velocity Molecular Stream in W51B: A Ring Structure with Compact H II Regions, MNRAS, 296, 863

Mufson, S. L. \& Liszt, H. S., 1979, The H II RegionMolecular Cloud Complex W51, ApJ, 232, 451

Myers, P. C., Dame, T. M., Thaddeus, P., Cohen, R. S., Silverberg, R. F., Dwek, E., \& Hauser, M. G., 1986, Molecular Clouds and Star Formation in the Inner Galaxy: A Comparison of CO, H II , and FarInfrared Surveys, ApJ, 301, 398

Nanda Kumar, M. S., Kamath, U. S., \& Davis, C. J., 2004, Embedded Star Clusters in the W51 Giant Molecular Cloud, MNRAS, 353, 1025

Palla, F. \& Stahler, S. W., 2000, Accelerating Star Formation in Clusters and Associations, ApJ, 540, 255

Panagia, N., 1973, Some Physical Parameters of EarlyType Stars, AJ, 78, 929

Reid, I. N., et al., 1991, The second Palomar Sky Survey, PASP, 103, 661

Schaller, G., Schaerer, D., Meynet, G., \& Maeder, A., 1992, New Grids of Stellar Models from 0.8 to 120 Solar Masses at $\mathrm{Z}=0.020$ and $\mathrm{Z}=0.001$, A\&AS, 96, 269

Sung, H., Bessell, M. S., \& Chun, M.-Y., 2004, The Initial Mass Function and Young Brown Dwarf Candidates in NGC 2264. I. The Initial Mass Function around S Monocerotis, AJ, 128, 1684

Testi, L., Palla, F. \& Natta, A., 1998, A Search for Clustering around Herbig Ae/Be Stars. II. Atlas of the Observed Sources, A\&AS, 133, 81

Wainscoat, R. J., Cohen, M., Volk, K., Walker, H. J., \& Schwartz, D. E., 1992, A Model of the 8-25 Micron Point Source Infrared Sky, ApJS, 83, 111

Wood, D. O. S. \& Churchwell, E., 1989, The Morphologies and Physical Properties of Ultracompact H II Regions, ApJS, 69, 831 